\title{
Patients with Advanced Ovarian Cancer Administered Oral Etoposide following Taxane as Maintenance Chemotherapy
}

\author{
Hiroaki Nagano Yasunari Tachibana Megumi Kawakami Mariko Ueno \\ Yoshihiro Morita Mitsue Muraoka Koichiro Takagi
}

Department of Obstetrics and Gynecology, Tokyo Women's Medical University, Medical Center East, Tokyo, Japan

\section{Key Words}

Oral etoposide · Paclitaxel · Docetaxel · Maintenance therapy · Chemotherapy · Ovarian cancer

\begin{abstract}
Introduction: The concept of maintenance therapy is one of the highly relevant approaches in the management of advanced ovarian cancer. The fundamental goal of maintenance therapy is to improve survival outcomes. We attempted to reinforce maintenance chemotherapy by adding oral etoposide following taxane administration. Cases: We retrospectively evaluated 14 patients with advanced ovarian cancer who had achieved clinically defined complete response to a primary platinum/taxane chemotherapy regimen and who were administered oral etoposide (50 mg/day $\times 21$ days per cycle monthly for 3-5 cycles) following paclitaxel or docetaxel administration as maintenance chemotherapy. With regard to oral etoposide toxicity, grade 2 oral mucositis and grade 3 anemia were observed in 1 patient each. Three to five cycles of etoposide were administered to all patients, though daily dosage was reduced to $25 \mathrm{mg}$ in 2 patients due to toxicity. The median progression-free survival was 43.5 months, the median overall survival was 86 months, and 5 -year overall survival was $77.1 \%$. Conclusion: The results from this ovarian cancer treatment evaluation suggest that oral etoposide may be administered safely following paclitaxel or docetaxel as maintenance chemotherapy. We expect this regimen to contribute to the improvement in the survival outcomes of patients with advanced ovarian cancer.

\section{KARGER}

Hiroaki Nagano

Department of Obstetrics and Gynecology

Tokyo Women's Medical University, Medical Center East

2-1-10, Nishiogu, Arakawa-ku, Tokyo 116-8567 (Japan)

E-Mail naganoog@dnh.twmu.ac.jp 
Nagano et al.: Patients with Advanced Ovarian Cancer Administered Oral Etoposide following Taxane as Maintenance Chemotherapy

\section{Introduction}

Advanced ovarian cancer is considered one of the most lethal gynecological cancers. The majority of patients with this form of cancer ultimately develop tumor recurrence and succumb to the disease despite achieving clinical remission after completion of initial treatment [1]. The concept of maintenance therapy is one of the highly relevant approaches in the management of advanced ovarian cancer. The fundamental goal of maintenance therapy is to improve survival outcomes, including progression-free survival (PFS), symptom-free survival, and overall survival (OS) [2].

Recently, the antiangiogenic agents bevacizumab and pazopanib were shown to significantly improve the time to subsequent disease progression when utilized as maintenance therapy in phase 3 studies for advanced ovarian cancer [3-5]. However, an improvement in OS was not observed in these studies, and because of the expense of molecular targeting agents, the cost-effectiveness of such therapies has become subject to scrutiny [6-8].

On the other hand, the taxane paclitaxel is the only agent that has been shown to improve PFS among several cytotoxic agents investigated. Administration of 12 monthly cycles of paclitaxel compared with 3 cycles revealed remarkable improvement of PFS, although adverse events, such as peripheral neuropathy, negatively impacted the results. The authors recommended that oncologists should discuss with appropriate patients with advanced ovarian cancer the results of the trial and the possible implications for their subsequent management to prolong the time to disease progression $[9,10]$.

Docetaxel, another taxane, has been confirmed to have equal efficacy and reduced neurotoxicity compared with paclitaxel when used with carboplatin as initial chemotherapy for ovaria cancer [11]. We recently published the results of a feasibility study focusing on docetaxel as an alternative maintenance chemotherapy agent used to reduce the risk of neurotoxicity associated with paclitaxel; however, the impact on survival outcomes was unclear [12].

Etoposide is a DNA topoisomerase II inhibitor, and oral etoposide has demonstrated substantial activity as second-line therapy for patients with platinum-resistant as well as paclitaxel-resistant advanced ovarian cancer [13-16]. Etoposide may be a potential candidate as a single agent used for maintenance therapy because it does not exhibit crossresistance to platinum or paclitaxel. However, to the best of our knowledge, there are no reliable reports of its use in a maintenance setting. The possibility that prolonged delivery of etoposide may confer a substantial risk of developing secondary leukemia is a matter of concern $[14,17,18]$. Hence, oral etoposide should be administered only for a relatively short time within the presumable safety limits.

To improve the survival of patients with advanced ovarian cancer, we attempted to reinforce maintenance chemotherapy by adding oral etoposide following taxane administration. This is a report of these patients.

\section{Cases}

From January 2008, we initiated maintenance chemotherapy with paclitaxel or docetaxel followed by sequential oral etoposide administration in patients with International Federation of Gynecology and Obstetrics (FIGO) stage III or IV ovarian, peritoneal, or tubal cancer. Eligibility criteria included (1) histologically confirmed epithelial ovarian, peritoneal, or tubal cancer; (2) macroscopic dissemination to more than a single site beyond the pelvis at initial surgery, and (3) no clinical evidence of disease after completion of initial treatment. Patients with a history of hypersensitivity to both paclitaxel and docetaxel were excluded. 
Nagano et al.: Patients with Advanced Ovarian Cancer Administered Oral Etoposide following Taxane as Maintenance Chemotherapy

We discussed this maintenance regimen including the cost and possible adverse events with our patients who met the eligibility criteria. After obtaining written informed consent from the patients who wished to implicate the regimen, they were administered the maintenance regimen as follows.

Following completion of the initial treatment, we administered paclitaxel (160-175 $\left.\mathrm{mg} / \mathrm{m}^{2}\right)$ or docetaxel $\left(70 \mathrm{mg} / \mathrm{m}^{2}\right)$ monthly for a maximum of 13 cycles. The actual number of cycles of taxane administration was entrusted to the discretion of the physicians; however, we aimed for at least 6 cycles. Docetaxel was administered to those who had already used this drug during initial treatment or who had symptoms of paclitaxel-induced peripheral neuropathy. Conversion from paclitaxel to docetaxel because of adverse events, including peripheral neuropathy, was allowed during maintenance therapy. Oral etoposide administration was planned as $50 \mathrm{mg} /$ day $\times 21$ days per cycle every 28 days for 3-5 cycles.

Between January 2008 and December 2011, 34 patients with FIGO stage III or IV ovarian, peritoneal, or tubal cancer were treated at our institute. Of these, 5 patients (14\%) had not achieved clinical complete response or relapsed during initial chemotherapy. Ten patients $(28 \%)$ had limited dissemination (i.e., only a single site or no dissemination beyond the pelvis at initial surgery). One patient had hypersensitivity to both paclitaxel and docetaxel in primary chemotherapy. Thus, 18 patients, all of whom agreed to receive the maintenance regimen, met the eligibility criteria and started maintenance taxane chemotherapy. Of these, 2 patients discontinued the scheduled taxane regimen because of disease progression and another 2 patients abandoned it due to adverse events (angina pectoris and interstitial pneumonitis). As a consequence, 14 patients ( 9 with ovarian cancer, 4 with peritoneal cancer, and 1 with tubal cancer) were administered oral etoposide sequentially following monthly taxane as maintenance chemotherapy.

The clinical characteristics of the patients are summarized in table 1 . The median patient age was 66.5 years. Twelve patients had FIGO stage III disease, and 2 had FIGO stage IV disease. Histological tumor types included high-grade serous adenocarcinoma in 12 patients and clear cell carcinoma in 2 patients. Lymph node metastasis was observed in 9 patients. Ten patients underwent primary debulking surgery, and 4 patients underwent interval debulking surgery following neoadjuvant chemotherapy. In 5 patients, including 2 subjected to interval debulking surgery, tumors were resected optimally (i.e., macroscopic complete resection). All patients received chemotherapy with carboplatin plus either paclitaxel or docetaxel as first-line regimen, although the number of cycles varied. All patients, including 7 suboptimal patients and 2 stage IV patients, achieved complete clinical response following initial treatment.

Regarding maintenance taxane, 4 patients received paclitaxel, 2 were switched to docetaxel due to neurotoxicity, and 8 received docetaxel. A median of 10 monthly taxane cycles (range: 6-13 cycles) were administered. For oral etoposide, 3-5 cycles (mostly 4 cycles) of etoposide were administered to all patients, although the daily dosage was reduced to $25 \mathrm{mg}$ in 2 patients due to toxicity.

Toxicities during our maintenance regimen were assessed according to the National Cancer Institute Common Terminology Criteria for Adverse Events (version 4) [19]. As shown in table 2, 10 (71.4\%) out of 14 patients experienced peripheral neuropathy during first-line or maintenance taxane administration. Although the majority of patients developed neuropathy while on the primary chemotherapy regimen, their symptoms were maintained during maintenance therapy, and all of them were able to continue taxane therapy. Grade 4 neutropenia was also observed frequently; however, no severe infectious events due to neutropenia were observed. 
Nagano et al.: Patients with Advanced Ovarian Cancer Administered Oral Etoposide following Taxane as Maintenance Chemotherapy

With regard to oral etoposide, oral mucositis was the most frequent adverse event observed, although the symptoms were mostly mild and well tolerable. The dose of etoposide was reduced to $25 \mathrm{mg} /$ day in only 1 patient because of grade 2 mucositis and diarrhea. In another patient, the dose was reduced on account of grade 2 nausea. Hematological toxicity was also generally mild, although grade 3 anemia was observed in 1 patient. No patient experienced secondary malignancy.

PFS was defined as the duration from the date of initial therapy (surgery or neoadjuvant chemotherapy) to the date of the first indication of disease progression or death, whichever occurred first. Data for patients who were alive without disease progression were censored as of the date of their last assessment. OS was calculated from the date of initial therapy to the date of death from any cause; data for patients still alive were censored at the date the patient was last known to be alive. The Kaplan-Meier method was applied to estimate PFS and OS curves. As of the most recent assessment, the median follow-up period for censored patients was 61 months (range: 50-99 months) from the initiation of treatment; 10 patients $(71.4 \%)$ exhibited disease progression, and $5(35.7 \%)$ died from their disease. The median PFS was 43.5 months (range: 24-92 months); the median OS for this patient population was 86 months (range: 43-99 months), and the 5-year OS rate was 77.1\%. Fig. 1 and fig. 2 display the PFS and OS of the study cohort. The 5-year OS rate for all 34 patients with advanced ovarian cancer in our institute treated during the same periods was $58.5 \%$.

\section{Discussion}

Despite the small number of cases, we mainly focused on two clinical observations. The first one was toxicity; oral etoposide could be administered safely following paclitaxel or docetaxel as maintenance chemotherapy. The observed etoposide toxicity was generally mild, although grade 2 oral mucositis and grade 3 anemia were observed in 1 patient each. Severe neutropenia due to etoposide was not observed in any patients. Rose et al. [14] reported that grade 3-4 neutropenia was the most frequent adverse effect of oral etoposide (observed in $45 \%$ of their cohort), when administered as a second-line therapy. The cause of milder hematotoxicities in our cohort is unclear; however, the patients' general condition, prior taxane maintenance therapy, or cycles of etoposide administration may have caused a difference in the hematotoxicities. Secondary leukemia is the most severe adverse event of etoposide [14, 17, 18]. Le Deley et al. [18] reported that the risk of developing secondary leukemia increases with the cumulative dose of etoposide, with a particularly high risk in patients receiving more than $6.0 \mathrm{~g} / \mathrm{m}^{2}$. Rose et al. [14] also reported that 1 patient $(0.53 \%)$ developed leukemia following 10 cycles of oral etoposide chemotherapy. We did not refer to another phase 1 study, because oral topotecan was administered sequentially with etoposide in the study [20]. Here, we limited the number of cycles of etoposide administration to $\leq 5$ in order to avoid the risk of developing secondary leukemia. The maximal cumulative dose of oral etoposide in this study was $4.0 \mathrm{~g} / \mathrm{m}^{2}$. The risk of developing secondary malignancy should be carefully evaluated in further studies, although we did not experience such an event in our cohort.

The second clinical observation was survival outcomes. The observed median PFS was 43.5 months and 5-year OS was $77.1 \%$, with a median follow-up period of 61 months from treatment initiation. These survival outcomes are significantly better than the data from other studies targeting similar patient populations. Complete resection of all macroscopic disease processes by primary debulking surgery has been shown to be the single most important independent prognostic factor in advanced ovarian cancer [21-23]. The complete 
Nagano et al.: Patients with Advanced Ovarian Cancer Administered Oral Etoposide following Taxane as Maintenance Chemotherapy

resection rate by primary debulking surgery in our cohort was $21 \%$, and there is no significant difference compared with the result $(19 \%)$ of the large-scale study targeting similar patient populations, in which the median OS was 29-30 months [24]. In general, patients with stage III disease have a 5-year survival of approximately 15-20\%, whereas patients with stage IV disease have a 5 -year survival of $<5 \%$ [25]. In more recent meta-analysis data, the 5-year OS rate for advanced ovarian cancer patients (including patients with stage II disease of $9 \%$ ) was $39.0 \%$ [23]. They indicated that approximately $22 \%$ of the patients with advanced ovarian cancer were refractory or relapsed within 6 months after completion of chemotherapy (platinum-resistant). In our cohort, 7 out of 34 patients with stage III or IV disease $(20.6 \%)$ were considered to be platinum-resistant. Ten patients with only limited or no dissemination were also excluded from the candidates for our maintenance therapy. Including them, the 5-year OS rate for all 34 patients with advanced ovarian cancer in our cohort (including 6 with stage IV disease) was $58.5 \%$. We believe that the improvement of survival outcome in the maintenance therapy group mostly contributed to the overall favorable results. We could not identify which amongst taxane and oral etoposide was more beneficial for this favorable survival outcome. At least, however, our results were not inferior when compared with the results of the other maintenance paclitaxel study with similar sample size, in which observed median PFS was 24 months [26]. As mentioned previously, the taxane paclitaxel is the only agent that has been shown to improve PFS among several cytotoxic agents investigated. However, another 'maintenance paclitaxel' study failed to confirm a favorable impact associated with prolonged paclitaxel administration in a similar clinical setting [27], and the role of 'maintenance paclitaxel therapy' is still controversial. It should be noted that the Gynecologic Oncology Group (GOG) has recently completed accrual on a phase III trial (GOG212), which aimed at evaluating the outcomes after 'maintenance paclitaxel therapy' [2]. Pending the results of that study, we should refrain from making any definite statement on the efficacy of maintenance chemotherapy, including the regimen described here.

This study was performed as a retrospective study with a small number of patients at a single institute, and we cannot make definite conclusions. Nevertheless, this is the first report of oral etoposide administration in a maintenance setting for advanced ovarian cancer, and we would like to announce these encouraging results for patients who have been suffering from this lethal gynecological cancer. We expect this regimen to contribute to the improvement in the survival outcomes of patients with advanced ovarian cancer. However, it is difficult to precisely distinguish whether these results were only because of coincidence or bias or have some degree of truth. It should also be remembered that these maintenance chemotherapy regimens remain experimental therapies for clinical studies until their role in the management of advanced ovarian cancer is better established.

\section{Statement of Ethics}

The authors have no ethical conflicts to disclose.

\section{Disclosure Statement}

The authors declare that they have no conflicts of interest. 
Nagano et al.: Patients with Advanced Ovarian Cancer Administered Oral Etoposide following Taxane as Maintenance Chemotherapy

\section{References}

1 Fleming GF, Ronnett BM, Seidmann J, Zaino RJ, Rubin SC: Epithelial ovarian cancer; in Barakat RR, Markman M, Randall ME (eds): Principles and Practice of Gynecologic Oncology, ed 5. Philadelphia, Lippincott Williams and Wilkins, 2009, pp 763-835.

2 Markman M: Maintenance chemotherapy in the management of epithelial ovarian cancer. Cancer Metastasis Rev 2015;34:11-17.

-3 Burger RA, Brady MF, Bookman MA, Fleming GF, Monk BJ, Huang H, et al: Incorporation of bevacizumab in the primary treatment of ovarian cancer. N Engl J Med 2011;365:2473-2483.

-4 Perren TJ, Swart AM, Pfisterer J, Ledermann JA, Pujade-Lauraine E, Kristensen G, et al: A phase 3 trial of bevacizumab in ovarian cancer. N Engl J Med 2011;365:2484-2496.

5 du Bois A, Floquet A, Kim JW, Rau J, del Campo JM, Friedlander M, et al: Incorporation of pazopanib in maintenance therapy of ovarian cancer. J Clin Oncol 2014;32:3374-3382.

6 Cohn DE, Kim KH, Resnick KE, O'Malley DM, Straughn JM Jr, et al: At what cost does a potential survival advantage of bevacizumab make sense for the primary treatment of ovarian cancer? A cost-effectiveness analysis. J Clin Oncol 2011;29:1247-1251.

7 Mehta DA, Hay JW: Cost-effectiveness of adding bevacizumab to first line therapy for patients with advanced ovarian cancer. Gynecol Oncol 2014;132:677-683.

8 Bookman MA: Do we have a new standard in suboptimal debulked disease? Ann Oncol 2013;24(suppl 10): $\mathrm{x} 37-\mathrm{x} 40$.

9 Markman M, Liu PY, Wilczynski S, Monk B, Copeland LJ, Alvarez RD, et al: Phase III randomized trial of 12 versus 3 months of maintenance paclitaxel in patients with advanced ovarian cancer after complete response to platinum and paclitaxel-based chemotherapy: a Southwest Oncology Group and Gynecologic Oncology Group trial. J Clin Oncol 2003;21:2460-2465.

10 Markman M, Liu PY, Moon J, Monk BJ, Copeland L, Wilczynski S, et al: Impact on survival of 12 versus 3 monthly cycles of paclitaxel $\left(175 \mathrm{mg} / \mathrm{m}^{2}\right)$ administered to patients with advanced ovarian cancer who attained a complete response to primary platinum-paclitaxel: follow-up of a Southwest Oncology Group and Gynecologic Oncology Group phase 3 trial. Gynecol Oncol 2009;114:195-198.

-11 Vassey PA, Jayson GC, Gordon A, Gabra H, Coleman R, Atkinson R, et al: Phase III randomized trial of docetaxel-carboplatin versus paclitaxel-carboplatin as first-line chemotherapy for ovarian carcinoma. J Natl Cancer Inst 2004;22:1682-1691.

12 Isonishi S, Suzuki M, Nagano H, Takagi K, Shimauchi M, Kawabata M, et al: A feasibility study on maintenance of docetaxel after paclitaxel-carboplatin chemotherapy in patients with advanced ovarian cancer. J Gynecol Oncol 2013;24:154-159.

-13 Seymour MT, Mansi JL, Gallagher CJ, Gore ME, Harper PG, Evans TRJ, et al: Protracted oral etoposide in epithelial ovarian cancer: a phase II study in patients with relapsed or platinum-resistant disease. Br J Cancer 1994;69:191-195.

14 Rose PG, Blessing JA, Mayer AR, Homesley HD: Prolonged oral etoposide as second-line therapy for platinum-resistant and platinum-sensitive ovarian carcinoma: a Gynecologic Oncology Group study. J Clin Oncol 1998;16:405-410.

15 Alici S, Saip P, Eralp Y, Aydiner A, Topuz E: Oral etoposide (VP16) in platinum-resistant epithelial ovarian cancer (EOC). Am J Clin Oncol 2003;26:358-362.

16 Kucukoner M, Isikdogan A, Yaman S, Gumusay O, Unal O, Ulas A, et al: Oral etoposide for platinum-resistant and recurrent epithelial ovarian cancer: a study by the Anatolian Society of Medical Oncology. Asian Pac J Cancer Prev 2012;13:3973-3976.

17 Yagita M, Ieki Y, Onishi R, Huang CL, Adachi M, Horiike S, et al: Therapy-related leukemia and myelodysplasia following oral administration of etoposide for recurrent breast cancer. Int J Oncol 1998;13:91-96.

18 Le Deley MC, Leblanc T, Shamsaldin A, Raquin MA, Lacour B, Sommelet D, et al: Risk of secondary leukemia after a solid tumor in childhood according to the dose of epipodophyllotoxins and anthracyclines: a casecontrol study by the Société Française d'Oncologie Pédiatrique. J Clin Oncol 2003;21:1074-1081.

19 Common Toxicity Criteria: version 4.0 [cited; available from: http://www.ctep.info.nih.gov].

-20 Rose PG, Markman M, Bell JG, Fusco NL: Sequential prolonged oral topotecan and prolonged oral etoposide as second-line therapy in ovarian or peritoneal carcinoma: a phase I Gynecologic Oncology Group study. Gynecol Oncol 2006;102:236-239.

-21 Bristow RE, Tomacruz RS, Armstrong DK, Trimble EL, Montz FJ: Survival effect of maximal cytoreductive surgery for advanced ovarian carcinoma during the platinum era: a meta-analysis. J Clin Oncol 2002;20:1248-1259.

22 Chi DS, Eisenhauer EL, Lang J, Huh J, Haddad L, Abu-Rustum NR, et al: What is the optimal goal of primary cytoreductive surgery for bulky stage IIIC epithelial ovarian carcinoma (EOC)? Gynecol Oncol 2006;103:559-564. 
Nagano et al.: Patients with Advanced Ovarian Cancer Administered Oral Etoposide following Taxane as Maintenance Chemotherapy

23 du Bois A, Reuss A, Pujade-Lauraine E, Harter P, Ray-Coquard I, Pfisterer J: Role of surgical outcome as prognostic factor in advanced epithelial ovarian cancer: a combined exploratory analysis of 3 prospectively randomized phase 3 multicenter trials. Cancer 2009;115:1234-1244.

24 Vergote I, Tropé CG, Amant F, Kristensen GB, Ehlen T, Johnson N, et al: Neoadjuvant chemotherapy or primary surgery in stage IIIC or IV ovarian cancer. N Engl J Med 2010;363:943-953.

-25 Heintz AP, Odicino F, Maisonneve P, Beller U, Benedet JL, Creasman WT, et al: Carcinoma of the ovary. Int J Gynecol Obstet 2003;83:135-166.

-26 Abaid LN, Goldstein BH, Micha JP, Rettenmaier MA, Brown JV 3rd, Markman M: Improved overall survival with 12 cycles of single-agent paclitaxel maintenance therapy following a complete response to induction chemotherapy in advanced ovarian carcinoma. Oncology 2010;78:389-393.

27 Pecorelli S, Favalli G, Gadducci A, Katsaros D, Panici PB, Carpi A, et al: Phase III trial of observation versus six courses of paclitaxel in patients with advanced epithelial ovarian cancer in complete response after six courses of paclitaxel/platinum-based chemotherapy: final results of the After-6 protocol 1. J Clin Oncol 2009;27:4642-4648. 


\section{Case Reports in Oncology}

Nagano et al.: Patients with Advanced Ovarian Cancer Administered Oral Etoposide following Taxane as Maintenance Chemotherapy

Table 1. Clinical characteristics

\begin{tabular}{|c|c|}
\hline \multicolumn{2}{|l|}{ Characteristic } \\
\hline Age, years & $\begin{array}{l}\text { median: } 66.5 \\
\text { (range: } 48-74 \text { ) }\end{array}$ \\
\hline \multicolumn{2}{|l|}{ Disease site } \\
\hline Ovary & $9(64.3)$ \\
\hline Peritoneum & $4(28.6)$ \\
\hline Tube & $1(7.1)$ \\
\hline \multicolumn{2}{|l|}{ Stage/debulking status } \\
\hline III, not macroscopic & $5(35.7)^{*}$ \\
\hline $\mathrm{III}, \leq 1 \mathrm{~cm}$ & $5(35.7)^{*}$ \\
\hline III, $>1 \mathrm{~cm}$ & $2(14.3)$ \\
\hline IV & $2(14.3)$ \\
\hline \multicolumn{2}{|l|}{ Histological type } \\
\hline Serous, high grade & $12(85.7)$ \\
\hline Clear cell & $2(14.3)$ \\
\hline \multicolumn{2}{|l|}{ Lymph node metastasis } \\
\hline Positive & $9(64.3)$ \\
\hline Negative & $5(35.7)$ \\
\hline \multicolumn{2}{|l|}{ Neoadjuvant chemotherapy } \\
\hline Yes & $4(28.6)$ \\
\hline No & $10(71.4)$ \\
\hline \multicolumn{2}{|l|}{ Maintenance taxane } \\
\hline Taxane cycles & $\begin{array}{l}\text { median: } 10 \\
\text { (range: } 6-13 \text { ) }\end{array}$ \\
\hline Paclitaxel & $4(28.6)$ \\
\hline Paclitaxel to docetaxel & $2(14.3)$ \\
\hline Docetaxel & $8(57.1)$ \\
\hline \multicolumn{2}{|l|}{ Etoposide cycles } \\
\hline 3 cycles & $3(21.4)$ \\
\hline 4 cycles & $9(64.3)^{* *}$ \\
\hline 5 cycles & $2(14.3)$ \\
\hline
\end{tabular}

Values are expressed as $\mathrm{n}(\%)$ unless indicated otherwise. * Including 2 patients with interval debulking surgery. ${ }^{* *}$ Including 2 patients in whom the daily dosage was reduced to $25 \mathrm{mg}$. 
Nagano et al.: Patients with Advanced Ovarian Cancer Administered Oral Etoposide following Taxane as Maintenance Chemotherapy

Table 2. Main adverse events of maintenance chemotherapy $(n=14)$

\begin{tabular}{|c|c|c|c|c|}
\hline & \multicolumn{4}{|l|}{ Grade* } \\
\hline & 1 & 2 & 3 & 4 \\
\hline \multicolumn{5}{|l|}{ Paclitaxel or docetaxel } \\
\hline Neutropenia & 0 & $1(7.1)$ & $3(21.4)$ & $10(71.4)$ \\
\hline Anemia & $6(42.9)$ & $8(57.1)$ & 0 & 0 \\
\hline Thrombocytopenia & $6(42.9)$ & 0 & 0 & 0 \\
\hline Peripheral neuropathy & $3(21.4)$ & $7(50.0)$ & 0 & 0 \\
\hline \multicolumn{5}{|l|}{ Oral etoposide } \\
\hline Neutropenia & $2(14.2)$ & $5(35.7)$ & $2(14.2)$ & 0 \\
\hline Anemia & $6(42.9)$ & $4(28.6)$ & $1(7.1)$ & 0 \\
\hline Oral mucositis & $5(35.7)$ & $1(7.1)$ & 0 & 0 \\
\hline Nausea & 0 & $1(7.1)$ & 0 & 0 \\
\hline Diarrhea & 0 & $1(7.1)$ & 0 & 0 \\
\hline
\end{tabular}

Values are expressed as $\mathrm{n}(\%) .{ }^{*}$ Graded using the National Cancer Institute Common Terminology Criteria for Adverse Events (version 4).

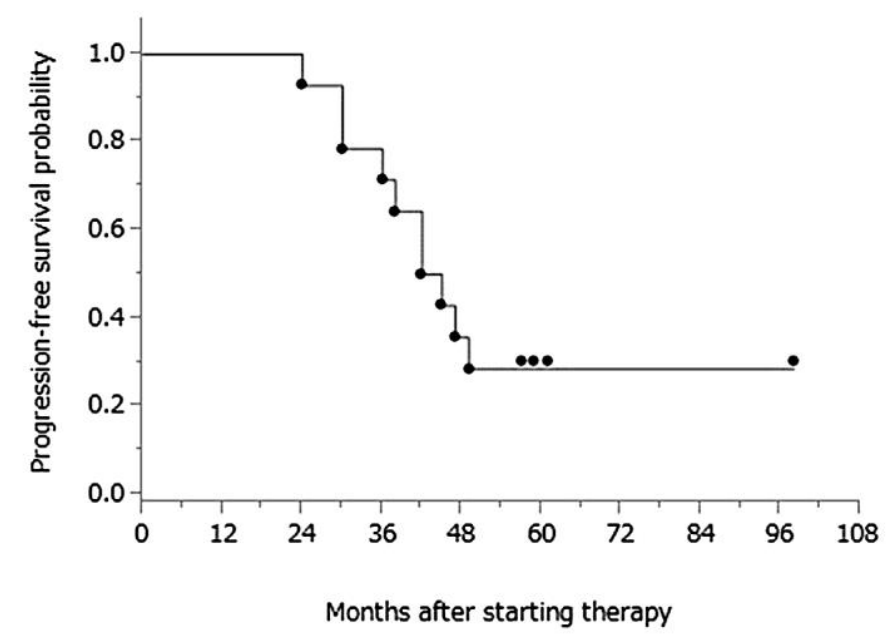

Fig. 1. PFS from treatment initiation. 
Nagano et al.: Patients with Advanced Ovarian Cancer Administered Oral Etoposide following Taxane as Maintenance Chemotherapy

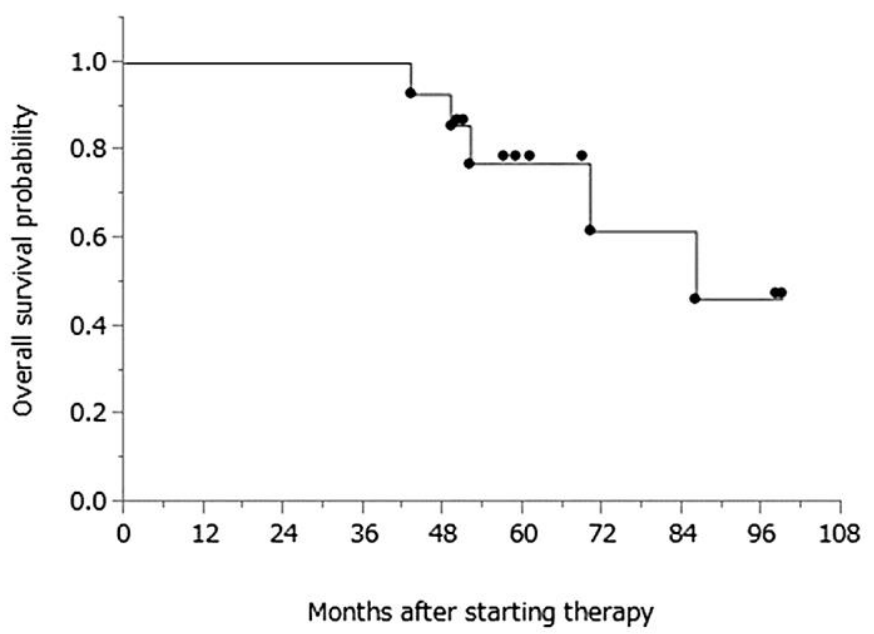

Fig. 2. OS with a median follow-up period of 61 months (range: 50-99 months) from treatment initiation for censored patients. 